PROCEEDINGS OF THE

AMERICAN MATHEMATICAL SOCIETY

Volume 139, Number 12, December 2011, Pages 4385-4400

S 0002-9939(2011)10852-9

Article electronically published on April 22, 2011

\title{
VARIOUS CHARACTERIZATIONS OF PRODUCT HARDY SPACE
}

\author{
JI LI, LIANG SONG, AND CHAOQIANG TAN
}

(Communicated by Michael T. Lacey)

\begin{abstract}
This article deals with the characterizations of Hardy space $H^{1}$ on $\mathbb{R}^{n} \times \mathbb{R}^{m}$ using different norms on distinct variables. This result can be applied to the boundedness of certain operators on $H^{1}\left(\mathbb{R}^{n} \times \mathbb{R}^{m}\right)$.
\end{abstract}

\section{INTRODUCTION}

The product Hardy space theory was developed by R. Gundy and E.M. Stein 7, S.-Y. Chang and R. Fefferman 1, 2, 4, 5, as well as J. Journé 11, 12. See [13, 15, 16 for some recent progress on multiparameter theory. As we all know, the space $H^{1}\left(\mathbb{R}^{n} \times \mathbb{R}^{m}\right)$ has a variety of different norms, in terms of maximal functions, square functions and Riesz transforms (see Theorem 3.4 of [13]).

Theorem A (Equivalent forms of $H^{1}$ norm). All the norms below are equivalent and can be used as a definition of $H^{1}\left(\mathbb{R}^{n} \times \mathbb{R}^{m}\right)$ :

$$
\left\|f^{+}\right\|_{L^{1}} \simeq\left\|f^{*}\right\|_{L^{1}} \simeq\|f\|_{L^{1}}+\|S(f)\|_{L^{1}} \simeq \sum_{i=0}^{n} \sum_{j=0}^{m}\left\|R_{1, i} R_{2, j} f\right\|_{L^{1}},
$$

where $S(f)$ is the Littlewood-Paley square function and we use $f^{+}$and $f^{*}$ to denote the vertical and nontangential maximal function, respectively, $R_{1, i}$ to denote the $i$ th Riesz transform for the first variable $x_{1} \in \mathbb{R}^{n}$ and $R_{2, j}$ the $j$ th Riesz transform for the second variable $x_{2} \in \mathbb{R}^{m}$, and the 0 th is the identity operator.

Moreover, $H^{1}\left(\mathbb{R}^{n} \times \mathbb{R}^{m}\right)$ adapts an atomic decomposition as follows. See Section 2 for the denotation.

Definition B. An $L^{2}\left(\mathbb{R}^{n} \times \mathbb{R}^{m}\right)$ function $a$ is called an $H^{1}\left(\mathbb{R}^{n} \times \mathbb{R}^{m}\right)$-atom if there exists an open set $\Omega$ with finite measure satisfying:

(1) $\operatorname{supp} a \subset \Omega$;

(2) $a$ has a further decomposition as $a=\sum_{R \in m(\Omega)} a_{R}$, where $a_{R}$ satisfy

(i) $\operatorname{supp} a_{R} \subset 3 R$;

Received by the editors July 10, 2010 and, in revised form, July 27, 2010 and October 20, 2010 .

2010 Mathematics Subject Classification. Primary 42B30, 42B35; Secondary 42B25, 46E40.

Key words and phrases. Multiparameter harmonic analysis, Hardy space.

The first author is supported by NNSF of China (11001275).

The second author is supported by NNSF of China (11001276) and is the corresponding author.

The third author is supported by FDYT of Guangdong (LYM08059).

(C)2011 American Mathematical Society 
(ii) $\int_{\mathbb{R}^{n}} a_{R}(x, y) d x=0, \quad \forall y \in \mathbb{R}^{m}, \quad \int_{\mathbb{R}^{m}} a_{R}(x, y) d y=0, \quad \forall x \in \mathbb{R}^{n}$; (3) $\|a\|_{2} \leq|\Omega|^{-1 / 2}$ and $\sum_{R \in m(\Omega)}\left\|a_{R}\right\|_{2}^{2} \leq|\Omega|^{-1}$.

Theorem C. $f \in H^{1}\left(\mathbb{R}^{n} \times \mathbb{R}^{m}\right)$ if and only if $f$ has a representation via atoms, i.e.

$$
f=\sum_{j} \lambda_{j} a_{j}
$$

where the $a_{j}$ are atoms as in Definition $B$ and $\sum_{j}\left|\lambda_{j}\right| \leq C\|f\|_{H^{1}\left(\mathbb{R}^{n} \times \mathbb{R}^{m}\right)}$.

In this paper, we develop several characterizations of Hardy space $H^{1}\left(\mathbb{R}^{n} \times \mathbb{R}^{m}\right)$ using different norms on distinct variables.

To begin with, we consider the following $(\mathcal{N}, S)$-function which appears as a nontangential maximal function on the first variable and as a Littlewood-Paley square function on the second variable. The notation $\Gamma\left(x_{1}\right)=\left\{\left(y_{1}, t_{1}\right) \in \mathbb{R}_{+}^{n+1}\right.$ : $\left.\left|x_{1}-y_{1}\right|<t_{1}\right\}$ denotes the standard cone with vertex $x_{1} \in \mathbb{R}^{n}$. Similarly, $\Gamma\left(x_{2}\right)=$ $\left\{\left(y_{2}, t_{2}\right) \in \mathbb{R}_{+}^{m+1}:\left|x_{2}-y_{2}\right|<t_{2}\right\}$ denotes the standard cone with vertex $x_{2} \in \mathbb{R}^{m}$ :

$$
f_{\mathcal{N}, S}\left(x_{1}, x_{2}\right):=\sup _{\left|y_{1}-x_{1}\right|<t_{1}}\left(\iint_{\Gamma\left(x_{2}\right)}\left|\phi_{t_{1}} \psi_{t_{2}} * f\left(y_{1}, y_{2}\right)\right|^{2} \frac{d y_{2} d t_{2}}{t_{2}^{m+1}}\right)^{1 / 2} .
$$

Here and throughout the whole paper, $\phi \in C^{\infty}\left(\mathbb{R}^{n}\right),|\phi(x)| \leq C(1+|x|)^{-n-1}$, $|\nabla \phi(x)| \leq C(1+|x|)^{-n-2}, \int \phi(x) d x=1$ and $\phi_{t_{1}}(x)=t_{1}^{-n} \phi\left(\frac{x}{t_{1}}\right), \psi \in C^{\infty}\left(\mathbb{R}^{m}\right)$, $|\psi(y)|$

$\leq C(1+|y|)^{-m-1},|\nabla \psi(y)| \leq C(1+|y|)^{-m-2}, \int \psi(y) d y=0, \int_{0}^{\infty}|\hat{\psi}(\xi t)|^{2} d t / t \leq C$ and $\psi_{t_{2}}(y)=t_{2}^{-m} \psi\left(\frac{y}{t_{2}}\right)$, where $t_{1}>0, t_{2}>0$ and $C$ is a positive constant.

Then we will prove that $H^{1}\left(\mathbb{R}^{n} \times \mathbb{R}^{m}\right)$ can be characterized in terms of the $(\mathcal{N}, S)$-function. To see this, define

$$
H_{\mathcal{N}, S}^{1}\left(\mathbb{R}^{n} \times \mathbb{R}^{m}\right):=\left\{f \mid f \in L^{1}\left(\mathbb{R}^{n} \times \mathbb{R}^{m}\right), f_{\mathcal{N}, S} \in L^{1}\left(\mathbb{R}^{n} \times \mathbb{R}^{m}\right)\right\}
$$

with the norm

$$
\|f\|_{H_{\mathcal{N}, S}^{1}\left(\mathbb{R}^{n} \times \mathbb{R}^{m}\right)}:=\|f\|_{L^{1}\left(\mathbb{R}^{n} \times \mathbb{R}^{m}\right)}+\left\|f_{\mathcal{N}, S}\right\|_{L^{1}\left(\mathbb{R}^{n} \times \mathbb{R}^{m}\right)} .
$$

We then have the first main result of this paper as follows.

Theorem 1.1. $H^{1}\left(\mathbb{R}^{n} \times \mathbb{R}^{m}\right) \simeq H_{\mathcal{N}, S}^{1}\left(\mathbb{R}^{n} \times \mathbb{R}^{m}\right)$.

Note that Theorem A implies that $H^{1}\left(\mathbb{R}^{n} \times \mathbb{R}^{m}\right)$ can be characterized by the product type nontangential maximal function or the Littlewood-Paley square function, which can be considered as the nontangential maximal function or the Littlewood-Paley square function (in the single parameter case) acting on both the first and second variables. Our result shows that one can also use the combination of the nontangential maximal function and the Littlewood-Paley square function (in the single parameter case) to give an equivalent characterization of $H^{1}\left(\mathbb{R}^{n} \times \mathbb{R}^{m}\right)$. Thus, we can further consider the combinations among the Littlewood-Paley $g$ function, vertical maximal function, as well as the nontangential maximal function and the Littlewood-Paley square function in the single parameter case. That is, we 
consider the $(\mathcal{N}, g)$-function and $(S, \mathcal{N})$-function as follows:

$$
\begin{aligned}
f_{\mathcal{N}, g}\left(x_{1}, x_{2}\right) & :=\sup _{\left|y_{1}-x_{1}\right|<t_{1}}\left(\int_{0}^{\infty}\left|\phi_{t_{1}} \psi_{t_{2}} * f\left(y_{1}, x_{2}\right)\right|^{2} \frac{d t_{2}}{t_{2}}\right)^{1 / 2} ; \\
f_{S, \mathcal{N}}\left(x_{1}, x_{2}\right) & :=\left(\iint_{\Gamma\left(x_{2}\right)} \sup _{\left|y_{1}-x_{1}\right|<t_{1}}\left|\phi_{t_{1}} \psi_{t_{2}} * f\left(y_{1}, y_{2}\right)\right|^{2} \frac{d y_{2} d t_{2}}{t_{2}^{m+1}}\right)^{1 / 2} .
\end{aligned}
$$

Then we define

$$
H_{\mathcal{N}, g}^{1}\left(\mathbb{R}^{n} \times \mathbb{R}^{m}\right):=\left\{f \mid f \in L^{1}\left(\mathbb{R}^{n} \times \mathbb{R}^{m}\right), f_{\mathcal{N}, g} \in L^{1}\left(\mathbb{R}^{n} \times \mathbb{R}^{m}\right)\right\}
$$

with the norm

$$
\|f\|_{H_{\mathcal{N}, g}^{1}\left(\mathbb{R}^{n} \times \mathbb{R}^{m}\right)}:=\|f\|_{L^{1}\left(\mathbb{R}^{n} \times \mathbb{R}^{m}\right)}+\left\|f_{\mathcal{N}, g}\right\|_{L^{1}\left(\mathbb{R}^{n} \times \mathbb{R}^{m}\right)},
$$

and

$$
H_{S, \mathcal{N}}^{1}\left(\mathbb{R}^{n} \times \mathbb{R}^{m}\right):=\left\{f \mid f \in L^{1}\left(\mathbb{R}^{n} \times \mathbb{R}^{m}\right), f_{S, \mathcal{N}} \in L^{1}\left(\mathbb{R}^{n} \times \mathbb{R}^{m}\right)\right\},
$$

with the norm

$$
\|f\|_{H_{S, \mathcal{N}}^{1}\left(\mathbb{R}^{n} \times \mathbb{R}^{m}\right)}:=\|f\|_{L^{1}\left(\mathbb{R}^{n} \times \mathbb{R}^{m}\right)}+\left\|f_{S, \mathcal{N}}\right\|_{L^{1}\left(\mathbb{R}^{n} \times \mathbb{R}^{m}\right)} .
$$

Similarly, we can define spaces $H_{g, \mathcal{N}}^{1}\left(\mathbb{R}^{n} \times \mathbb{R}^{m}\right), H_{+, S}^{1}\left(\mathbb{R}^{n} \times \mathbb{R}^{m}\right), H_{+, g}^{1}\left(\mathbb{R}^{n} \times\right.$ $\left.\mathbb{R}^{m}\right), H_{S,+}^{1}\left(\mathbb{R}^{n} \times \mathbb{R}^{m}\right)$ and $H_{g,+}^{1}\left(\mathbb{R}^{n} \times \mathbb{R}^{m}\right)$ in the norms given by the $L^{1}$ norm of the corresponding function plus the $L^{1}$ norm of the function itself, e.g.

$$
\|f\|_{H_{+, S}^{1}\left(\mathbb{R}^{n} \times \mathbb{R}^{m}\right)}:=\|f\|_{L^{1}\left(\mathbb{R}^{n} \times \mathbb{R}^{m}\right)}+\left\|f_{+, S}\right\|_{L^{1}\left(\mathbb{R}^{n} \times \mathbb{R}^{m}\right)},
$$

where $f_{+, S}\left(x_{1}, x_{2}\right)=: \sup _{t_{1}>0}\left(\iint_{\Gamma\left(x_{2}\right)}\left|\phi_{t_{1}} \psi_{t_{2}} * f\left(x_{1}, y_{2}\right)\right|^{2} \frac{d y_{2} d t_{2}}{t_{2}^{m+1}}\right)^{1 / 2}$.

Now we state the second main result of this paper as follows.

Theorem 1.2. Let all the notation be the same as above. Then we have

$$
\begin{aligned}
& H^{1}\left(\mathbb{R}^{n} \times \mathbb{R}^{m}\right) \simeq H_{\mathcal{N}, g}^{1}\left(\mathbb{R}^{n} \times \mathbb{R}^{m}\right) \simeq H_{S, \mathcal{N}}^{1}\left(\mathbb{R}^{n} \times \mathbb{R}^{m}\right) \simeq H_{g, \mathcal{N}}^{1}\left(\mathbb{R}^{n} \times \mathbb{R}^{m}\right) \\
& \simeq H_{+, S}^{1}\left(\mathbb{R}^{n} \times \mathbb{R}^{m}\right) \simeq H_{+, g}^{1}\left(\mathbb{R}^{n} \times \mathbb{R}^{m}\right) \simeq H_{S,+}^{1}\left(\mathbb{R}^{n} \times \mathbb{R}^{m}\right) \simeq H_{g,+}^{1}\left(\mathbb{R}^{n} \times \mathbb{R}^{m}\right) .
\end{aligned}
$$

The paper is organized as follows. In Section 2, we introduce some definitions and preliminary results. In Section 3, we prove Theorem 1.1 using two approaches. In the last section we prove Theorem 1.2

\section{Preliminaries}

For any $x_{2} \in \mathbb{R}^{m}$, define $\mathbb{B}\left(x_{2}\right):=\left\{F_{t_{2}, y_{2}}\left(y_{1}\right): t_{2}>0, y_{1} \in \mathbb{R}^{n}, y_{2} \in \Gamma\left(x_{2}\right)\right\}$ with the norm

$$
\left\|F_{t_{2}, y_{2}}\left(y_{1}\right)\right\|_{\mathbb{B}\left(x_{2}\right)}=\left(\iint_{\Gamma\left(x_{2}\right)}\left|F_{t_{2}, y_{2}}\left(y_{1}\right)\right|^{2} \frac{d y_{2} d t_{2}}{t_{2}^{m+1}}\right)^{1 / 2} .
$$

Next, we use $*_{2}$ to denote the convolution on the second variable; that is,

$$
f *_{2} g(x, y)=\int_{\mathbb{R}^{m}} f(z) g(x, y-z) d z
$$

for any functions $f$ on $\mathbb{R}^{m}$ and $g$ on $\mathbb{R}^{n} \times \mathbb{R}^{m}$. Similarly, we can define the convolution $*_{1}$ on the first variable.

Now we recall Journé's covering lemma ([10]) as follows. 
Suppose $\Omega \subset \mathbb{R}^{n} \times \mathbb{R}^{m}$ is open of finite measure. Denote by $m(\Omega)$ the maximal dyadic subrectangles of $\Omega$. Let $m_{1}(\Omega)$ denote those dyadic subrectangles $R \subseteq$ $\Omega, R=I \times J$ that are maximal in the $x_{1}$ direction. In other words if $S=I^{\prime} \times J \supseteq R$ is a dyadic subrectangle of $\Omega$, then $I=I^{\prime}$. Define $m_{2}(\Omega)$ similarly.

Lemma 2.1. For any $R=I \times J \in m_{1}(\Omega)$, we set $\gamma_{1}(R)=\sup _{\substack{I \subset l \\ l \times J \subset \Omega^{*}}}|l| /|I|$, where $\Omega^{*}=\left\{x \in \mathbb{R}^{n+m}, \mathcal{M}\left(\chi_{\Omega}\right)(x)>\frac{1}{2}\right\}$. Define $\gamma_{2}$ similarly. Then for any $\delta>0$,

$$
\sum_{R \in m_{2}(\Omega)}|R| \gamma_{1}^{-\delta}(R) \leq c_{\delta}|\Omega| \quad \text { and } \sum_{R \in m_{1}(\Omega)}|R| \gamma_{2}^{-\delta}(R) \leq c_{\delta}|\Omega|,
$$

where $c_{\delta}$ is a constant depending only on $\delta$, not on $\Omega$.

Moreover, we need the following tent space theory which has been studied by $[\underline{3}$. $\mathbb{R}_{+}^{n+1}$ will denote the usual upper half-space in $\mathbb{R}^{n+1}$. If $O$ is an open subset of $\mathbb{R}^{n}$, then the "tent" over $O$, denoted by $\widehat{O}$, is given as $\widehat{O}=\left\{(x, t) \in \mathbb{R}_{+}^{n+1}: B(x, t) \subset O\right\}$. For any function $f(y, t)$ defined on $\mathbb{R}_{+}^{n+1}$ we will denote

$$
\mathcal{A}(f)(x)=\left(\iint_{\Gamma(x)}|f(y, t)|^{2} \frac{d y d t}{t^{n+1}}\right)^{1 / 2} .
$$

As in [3], the "tent space" $T_{2}^{p}$ is defined as the space of functions $f$ such that $\mathcal{A}(f) \in L^{p}\left(\mathbb{R}^{n}\right)$, when $p<\infty$. The resulting equivalence classes are then equipped with the norm $\|f\|_{T_{2}^{p}}=\|\mathcal{A}(f)\|_{p}$. We recall that a $T_{2}^{1}$ atom is a function $a(x, t)$ supported in $\widehat{Q}$ (for some cube $Q \subset \mathbb{R}^{n}$ ), with $\int_{\widehat{Q}}|a(x, t)|^{2} d x d t / t \leq 1 /|Q|$.

Proposition 2.2. Every element $F \in T_{2}^{1}\left(\mathbb{R}_{+}^{n+1}\right)$ can be written as $F=\sum_{j} \lambda_{j} a_{j}$, where the $a_{j}$ are $T_{2}^{1}$ atoms, and $\sum_{j}\left|\lambda_{j}\right| \leq C\|f\|_{T_{2}^{1}}$.

In the following Lemma 2.3 (see [14] for the proof), we shall assume that $\Phi \in$ $C_{0}^{1}\left(\mathbb{R}^{n}\right)$ is nonnegative, radial and nonincreasing, $\Phi=1$ on $B(0,1 / 2)$, supp $\Phi \subset$ $B(0,1)$ and $\int \Phi(x) d x=1$. We may sometimes use capital letters to denote points in $\mathbb{R}_{+}^{n+1}$, for example, $X=(x, t)$, and set $u(x, t)=P_{t} f(x)$,

$$
\nabla_{X} u(X)=\left(\nabla_{x} u, \partial_{t} u\right) \text { and }\left|\nabla_{X} u\right|^{2}=\left|\nabla_{x} u\right|^{2}+\left|\partial_{t} u\right|^{2} .
$$

Lemma 2.3. For every $f, g \in L^{2}\left(\mathbb{R}^{n}\right)$, we have

$$
\begin{aligned}
\iint_{\mathbb{R}_{+}^{n+1}}\left|t \nabla_{X} u(x, t)\right|^{2}\left|\Phi_{t} * g(x)\right|^{2} \frac{d x d t}{t} & \\
& \leq \int_{\mathbb{R}^{n}}|f(x)|^{2}|g(x)|^{2} d x+\iint_{\mathbb{R}_{+}^{n+1}}|u(x, t)|^{2}\left|\Psi_{t} * g(x)\right|^{2} \frac{d x d t}{t},
\end{aligned}
$$

where $\Psi$ is a vector-valued function with the same support as $\Phi$ and mean value 0 .

\section{Proof of Theorem 1.1}

First we point out that the definition of the space $H_{\mathcal{N}, S}^{1}\left(\mathbb{R}^{n} \times \mathbb{R}^{m}\right)$ is independent of the choices of functions $\phi$ and $\psi$ which satisfy the conditions as in (1.1).

In fact, we first fix a function $\psi$ satisfying the conditions as in (1.1), and then we prove that the definition of $H_{\mathcal{N}, S}^{1}\left(\mathbb{R}^{n} \times \mathbb{R}^{m}\right)$ is independent of the choices of functions 
$\phi$. To see this, we set $F_{t_{2}, y_{2}}\left(y_{1}\right)=\psi_{t_{2}} *_{2} f\left(y_{1}, y_{2}\right)$, where $f \in L^{1}\left(\mathbb{R}^{n} \times \mathbb{R}^{m}\right)$. Then, define

$$
\begin{aligned}
& M_{\phi}(f)\left(x_{1}, x_{2}\right):=\sup _{t_{1}>0}\left\|F_{t_{2}, y_{2}} *_{1} \phi_{t_{1}}\left(x_{1}\right)\right\|_{\mathbb{B}\left(x_{2}\right)}, \\
& F^{*}\left(x_{1}, x_{2}\right):=\sup _{\left|x_{1}-y_{1}\right|<t_{1}}\left\|F_{t_{2}, y_{2}} *_{1} P_{t_{1}}\left(y_{1}\right)\right\|_{\mathbb{B}\left(x_{2}\right)},
\end{aligned}
$$

where $P_{t_{1}}$ is the Poisson kernel on $\mathbb{R}^{n}$. Here and throughout this section, we use $\|\cdot\|_{\mathbb{B}\left(x_{2}\right)}$ to denote the vector-valued norm defined as in (2.1). It is easy to see that $P_{t_{1}}$ satisfies all conditions as $\phi$ in (1.1). Moreover, let $\mathcal{F}=\left\{\|\cdot\|_{\alpha, \beta}\right\}$ be any finite collection of seminorms on the test function space $\mathcal{S}$, where $\|\cdot\|_{\alpha, \beta}$ is given by $\|\phi\|_{\alpha, \beta}=\sup _{x \in \mathbb{R}^{n}}\left|x^{\alpha} \partial_{x}^{\beta} \phi(x)\right|$ and $\alpha$ and $\beta$ are $n$-tuples of natural numbers. Define $\mathcal{S}_{\mathcal{F}}=\left\{\phi \in \mathcal{S}:\|\phi\|_{\alpha, \beta} \leq 1\right.$, for all $\left.\|\cdot\|_{\alpha, \beta} \in \mathcal{F}\right\}$ and

$$
M_{\mathcal{F}}(f)\left(x_{1}, x_{2}\right)=\sup _{\phi \in \mathcal{S}_{\mathcal{F}}} M_{\phi}(f)\left(x_{1}, x_{2}\right) .
$$

Then we have the following result:

Proposition 3.1. Let $f \in L^{1}\left(\mathbb{R}^{n} \times \mathbb{R}^{m}\right)$. Then the following conditions are equivalent:

(i) There is a $\phi \in \mathcal{S}$ with $\int \phi d x \neq 0$ so that $M_{\phi}(f) \in L^{1}\left(\mathbb{R}^{n} \times \mathbb{R}^{m}\right)$.

(ii) There is a collection $\mathcal{F}$ so that $M_{\mathcal{F}}(f) \in L^{1}\left(\mathbb{R}^{n} \times \mathbb{R}^{m}\right)$.

(iii) $F^{*} \in L^{1}\left(\mathbb{R}^{n} \times \mathbb{R}^{m}\right)$.

We can obtain this proposition by following the proof of Theorem 1 as in Chapter $3, \S 1.2$, 17 with only minor modifications.

Proposition 3.1 implies that for any fixed $\psi$, the definition of $H_{\mathcal{N}, S}^{1}\left(\mathbb{R}^{n} \times \mathbb{R}^{m}\right)$ is independent of the choices of the functions $\phi$. Then in the proofs of Propositions 3.2 and 3.3. we fix a function $\psi$ and then use the Poisson kernel in the definition of $H_{\mathcal{N}, S}^{1}\left(\mathbb{R}^{n} \times \mathbb{R}^{m}\right)$ instead of $\phi$ to prove Theorem 1.1. This yields that the definition of $H_{\mathcal{N}, S}^{1}\left(\mathbb{R}^{n} \times \mathbb{R}^{m}\right)$ is also independent of the choices of $\psi$.

Now we will prove Theorem 1.1 by showing the following two propositions.

Proposition 3.2. $H^{1}\left(\mathbb{R}^{n} \times \mathbb{R}^{m}\right) \subseteq H_{\mathcal{N}, S}^{1}\left(\mathbb{R}^{n} \times \mathbb{R}^{m}\right)$.

Proposition 3.3. $H_{\mathcal{N}, S}^{1}\left(\mathbb{R}^{n} \times \mathbb{R}^{m}\right) \subseteq H^{1}\left(\mathbb{R}^{n} \times \mathbb{R}^{m}\right)$.

Proof of Proposition 3.2. In this part, we give two approaches: one relies on the atomic decomposition for the product Hardy space $H^{1}\left(\mathbb{R}^{n} \times \mathbb{R}^{m}\right)$ (see [2, 8, for the idea); the other is due to the result of C. Fefferman and E.M. Stein ([6]), using the distribution inequality.

Method 1 (via atomic decomposition). Let $f(x)=\sum_{j} \lambda_{j} a_{j}(x)$, where the $a_{j}$ 's are product atoms and $\sum_{j=0}^{\infty}\left|\lambda_{j}\right|<\infty$. We note that the operator $T_{\mathcal{N}, S}: f \mapsto f_{\mathcal{N}, S}$ is bounded on $L^{2}\left(\mathbb{R}^{n} \times \mathbb{R}^{m}\right)$, where $f_{\mathcal{N}, S}$ was defined in (1.1). Recently, Han et al. have showed that a linear operator $T$, which is bounded on $L^{2}\left(\mathbb{R}^{n} \times \mathbb{R}^{m}\right)$, is bounded from $H^{1}\left(\mathbb{R}^{n} \times \mathbb{R}^{m}\right)$ to $L^{1}\left(\mathbb{R}^{n} \times \mathbb{R}^{m}\right)$ if $T$ is uniformly bounded on all product atoms in $L^{1}\left(\mathbb{R}^{n} \times \mathbb{R}^{m}\right)$. See Theorem 1.3 of 9 . The proof is also applicable to sublinear operators. Thus, to prove Proposition [3.2] it suffices to show that there exists some constant $C$ such that for each product atom $a$,

$$
\left\|a_{\mathcal{N}, S}\right\|_{L^{1}\left(\mathbb{R}^{n} \times \mathbb{R}^{m}\right)} \leq C .
$$


Assume that $a=\sum_{R \in m(\Omega)} a_{R}$ is a product atom supported in some open set $\Omega$ of $\mathbb{R}^{n+m}$ with finite measure. For any $R=I \times J \in m(\Omega)$, let $\ell(I), \ell(J)$ be the side-lengths of cubes $I$ and $J$, and $I^{\prime}$ be the longest dyadic interval containing $I$ so that $I^{\prime} \times J \subset \Omega^{*}=\left\{x \in \mathbb{R}^{n+m}: \mathcal{M}_{s}\left(\chi_{\Omega}\right)(x)>1 / 2\right\}$. Then $I^{\prime} \times J$ is in $m_{1}\left(\Omega^{*}\right)$, and let $J^{\prime}$ be the longest dyadic interval so that $J^{\prime} \supseteq J$ and $I^{\prime} \times J^{\prime} \subset \Omega^{* *}$, where $\Omega^{* *}=\left\{x \in \mathbb{R}^{n+m}: \mathcal{M}_{s}\left(\chi_{\Omega}^{*}\right)(x)>1 / 2\right\}$. Let $\widetilde{R}$ be the 10 -fold dilate of $I^{\prime} \times J^{\prime}$ concentric with $I^{\prime} \times J^{\prime}$. Clearly, the boundedness of the strong maximal function shows that $|\bigcup \widetilde{R}| \leq c\left|\Omega^{* *}\right| \leq c\left|\Omega^{*}\right| \leq c|\Omega|$. We have

$$
\int_{\bigcup \widetilde{R}} a_{\mathcal{N}, S}(x) d x \leq C|\bigcup \widetilde{R}|^{1 / 2}\left\|a_{\mathcal{N}, S}\right\|_{2} \leq C|\Omega|^{1 / 2}\left\|a_{\mathcal{N}, S}\right\|_{2} .
$$

We claim that $\left\|a_{\mathcal{N}, S}\right\|_{2} \leq C|\Omega|^{-1 / 2}$. In fact, observe that for each $\left|x_{1}-y_{1}\right|<t_{1}$, $y_{2} \in \mathbb{R}^{m}$,

$$
\left|\phi_{t_{1}} *_{1} f\left(y_{1}, y_{2}\right)\right| \leq C \mathcal{M}^{(1)}\left(f\left(\cdot, y_{2}\right)\right)\left(x_{1}\right),
$$

where we use $\mathcal{M}^{(1)}$ to denote the Hardy-Littlewood maximal operator on the first variable. By using the $L^{2}$-boundedness of $\mathcal{M}^{(1)}$ and the square function, we obtain

$$
\begin{aligned}
\iint & \left(a_{\mathcal{N}, S}\left(x_{1}, x_{2}\right)\right)^{2} d x_{1} d x_{2} \\
& \leq C \iiint \int_{\Gamma\left(x_{2}\right)}\left(\mathcal{M}^{(1)}\left(\psi_{t_{2}} *_{2} a\left(\cdot, y_{2}\right)\right)\left(x_{1}\right)\right)^{2} \frac{d y_{2} d t_{2}}{t_{2}^{m+1}} d x_{1} d x_{2} \\
& \leq C \iiint \int_{\Gamma\left(x_{2}\right)}\left|\psi_{t_{2}} *_{2} a\left(x_{1}, y_{2}\right)\right|^{2} \frac{d y_{2} d t_{2}}{t_{2}^{m+1}} d x_{1} d x_{2} \\
& \leq C\|a\|_{2} \leq C|\Omega|^{-1 / 2} .
\end{aligned}
$$

Therefore, we have proved $\int_{\cup \widetilde{R}} a_{\mathcal{N}, S}(x) d x \leq C$. It suffices to prove

$$
\int_{(\cup \widetilde{R})^{c}} a_{\mathcal{N}, S}(x) d x \leq C .
$$

One writes

$$
\begin{aligned}
\int_{(\bigcup \widetilde{R})^{c}} a_{\mathcal{N}, S}(x) d x & \leq \sum_{R \in m(\Omega)} \int_{(\widetilde{R})^{c}}\left(a_{R}\right)_{\mathcal{N}, S}(x) d x \\
& \leq \sum_{R \in m(\Omega)} \int_{x_{1} \notin 10 I^{\prime}}\left(a_{R}\right)_{\mathcal{N}, S}(x) d x+\int_{x_{2} \notin 10 J^{\prime}}\left(a_{R}\right)_{\mathcal{N}, S}(x) d x \\
& =: \quad \mathrm{D}+\mathrm{E} .
\end{aligned}
$$

We first estimate the term D. Observe that

$$
\mathrm{D}=\sum_{R \in m(\Omega)}\left(\int_{x_{1} \notin 10 I^{\prime}} \int_{x_{2} \in 10 J}+\int_{x_{1} \notin 10 I^{\prime}} \int_{x_{2} \notin 10 J}\right)\left(a_{R}\right)_{\mathcal{N}, S}(x) d x=: \mathrm{D}_{1}+\mathrm{D}_{2} .
$$

Denote $F_{t_{2}, y_{2}}\left(y_{1}\right):=\psi_{t_{2}} *_{2} a_{R}\left(y_{1}, y_{2}\right)$. By Hölder's inequality,

$$
\mathrm{D}_{1} \leq C \sum_{R \in m(\Omega)}|J|^{1 / 2} \int_{x_{1} \notin 10 I^{\prime}}\left(\int \sup _{\left|x_{1}-y_{1}\right|<t_{1}}\left\|\phi_{t_{1}} * F_{t_{2}, y_{2}}\left(y_{1}\right)\right\|_{\mathbb{B}\left(x_{2}\right)}^{2} d x_{2}\right)^{1 / 2} d x_{1} .
$$

Denote the center of $I$ and $J$ by $x_{I}$ and $x_{J}$, respectively. By noting that $\left|x_{1}-y_{1}\right|<t_{1}, z_{1} \in 3 I$ and $x_{1} \notin 10 I^{\prime}$, we can apply the cancellation of atoms 
(i.e. (ii) of (2) in Definition B) to obtain

$$
\begin{aligned}
\left|\phi_{t_{1}} \psi_{t_{2}} *\left(a_{R}\right)\left(y_{1}, y_{2}\right)\right| & =\left|\int_{3 I}\left(\phi_{t_{1}}\left(y_{1}-z_{1}\right)-\phi_{t_{1}}\left(y_{1}-x_{I}\right)\right) \psi_{t_{2}} *_{2}\left(a_{R}\right)\left(z_{1}, y_{2}\right) d z_{1}\right| \\
& \leq C \frac{t_{1}}{\left(t_{1}+\left|x_{1}-x_{I}\right|\right)^{n+1}}\left(1 \wedge \frac{\ell(I)}{t_{1}}\right) \int_{3 I}\left|\psi_{t_{2}} *_{2} a_{R}\left(z_{1}, y_{2}\right)\right| d z_{1} \\
& \leq C \frac{\ell(I)}{\left|x_{1}-x_{I}\right|^{n+1}}|I|^{1 / 2}\left(\int_{3 I}\left|\psi_{t_{2}} *_{2} a_{R}\left(z_{1}, y_{2}\right)\right|^{2} d z_{1}\right)^{1 / 2},
\end{aligned}
$$

where $\left(1 \wedge\left(\ell(I) / t_{1}\right)\right):=\min \left(1, \ell(I) / t_{1}\right)$. By substituting (3.6) back into (3.5), we have

$$
\begin{aligned}
\mathrm{D}_{1} & \leq C \sum_{R \in m(\Omega)}|R|^{1 / 2} \int_{x_{1} \notin 10 I^{\prime}} \frac{\ell(I)}{\left|x_{1}-x_{I}\right|^{n+1}}\left(\iint\left|a_{R}\left(z_{1}, y_{2}\right)\right|^{2} d z_{1} d y_{2}\right)^{1 / 2} d x_{1} \\
& \leq C \sum_{R \in m(\Omega)}|R|^{1 / 2} \frac{\ell(I)}{\ell\left(I^{\prime}\right)}\left\|a_{R}\right\|_{2} \\
& \leq C
\end{aligned}
$$

where in the last inequality we have used Hölder's inequality and Lemma 2.1

Consider the term $\mathrm{D}_{2}$. By $\int a_{R}\left(z_{1}, z_{2}\right) d z_{1}=0$ and $\int a_{R}\left(z_{1}, z_{2}\right) d z_{2}=0$, we have

$$
\begin{aligned}
& \left|\phi_{t_{1}} \psi_{t_{2}} * a_{R}\left(y_{1}, y_{2}\right)\right| \\
& =\left|\iint\left(\phi_{t_{1}}\left(y_{1}-z_{1}\right)-\phi_{t_{1}}\left(y_{1}-x_{I}\right)\right)\left(\psi_{t_{1}}\left(y_{2}-z_{2}\right)-\psi_{t_{2}}\left(y_{2}-x_{J}\right)\right) a_{R}\left(z_{1}, z_{2}\right) d z_{1} d z_{2}\right| \\
& \leq C \frac{t_{1}}{\left(t_{1}+\left|x_{1}-x_{I}\right|\right)^{n+1}}\left(1 \wedge \frac{\ell(I)}{t_{1}}\right) \frac{t_{2}}{\left(t_{2}+\left|x_{2}-x_{J}\right|\right)^{m+1}}\left(1 \wedge \frac{\ell(J)}{t_{2}}\right)\left\|a_{R}\right\|_{2}|R|^{1 / 2} \\
& \leq C \frac{\ell(I)}{\left|x_{1}-x_{I}\right|^{n+1}} \frac{t_{2}^{1 / 2}}{\left(t_{2}+\left|x_{2}-x_{J}\right|\right)^{m+1 / 2}}\left(1 \wedge \frac{\ell(J)}{t_{2}}\right)\left\|a_{R}\right\|_{2}|R|^{1 / 2} .
\end{aligned}
$$

Therefore,

$$
\begin{aligned}
\mathrm{D}_{2} \leq & C \sum_{R \in m(\Omega)}\left\|a_{R}\right\|_{2}|R|^{1 / 2} \int_{x_{1} \notin 10 I^{\prime}} \int_{x_{2} \notin 10 J} \frac{\ell(I)}{\left|x_{1}-x_{I}\right|^{n+1}} \\
& \times\left(\int_{0}^{\infty} \frac{t_{2}}{\left(t_{2}+\left|x_{2}-x_{J}\right|\right)^{2 m+1}}\left(1 \wedge \frac{\ell(J)}{t_{2}}\right)^{2} \frac{d t_{2}}{t_{2}}\right)^{1 / 2} d x_{2} d x_{1} \\
\leq & C \sum_{R \in m(\Omega)}\left\|a_{R}\right\|_{2}|R|^{1 / 2} \int_{x_{1} \notin 10 I^{\prime}} \int_{x_{2} \notin 10 J} \frac{\ell(I)}{\left|x_{1}-x_{I}\right|^{n+1}} \frac{\ell(J)^{1 / 2}}{\left|x_{2}-x_{J}\right|^{m+1 / 2}} d x_{2} d x_{2} \\
\leq & C \sum_{R \in m(\Omega)}\left\|a_{R}\right\|_{2}|R|^{1 / 2} \frac{\ell(I)}{\ell\left(I^{\prime}\right)} \leq C,
\end{aligned}
$$

where the last inequality follows from Hölder's inequality and Lemma 2.1.

We then estimate the term E. Observe that

$$
\mathrm{E}=\sum_{R \in m(\Omega)}\left(\int_{x_{1} \in 10 I} \int_{x_{2} \notin 10 J^{\prime}}+\int_{x_{1} \notin 10 I} \int_{x_{2} \notin 10 J^{\prime}}\right)\left(a_{R}\right)_{\mathcal{N}, S}(x) d x=: \mathrm{E}_{1}+\mathrm{E}_{2} .
$$


We only estimate $E_{1}$ since $E_{2}$ can be estimated by the similar argument to that of $\mathrm{D}_{2}$. Applying Hölder's inequality, we have

$\mathrm{E}_{1} \leq C|I|^{1 / 2} \sum_{R \in m(\Omega)} \int_{x_{2} \notin 10 J^{\prime}}\left(\int_{x_{1} \in 10 I} \sup _{\left|x_{1}-y_{1}\right|<t_{1}}\left\|\phi_{t_{1}} * F_{t_{2}, y_{2}}\left(y_{1}\right)\right\|_{\mathbb{B}\left(x_{2}\right)}^{2} d x_{1}\right)^{1 / 2} d x_{2}$.

Observe that $\left|\phi_{t_{1}} \psi_{t_{2}} * a_{R}\left(y_{1}, y_{2}\right)\right| \leq \mathcal{M}^{(1)}\left(\psi_{t_{2}} * a_{R}\left(\cdot, y_{2}\right)\right)\left(x_{1}\right)$ whenever $\left|x_{1}-y_{1}\right|<t_{1}$. This, together with the $L^{2}$-boundedness of $\mathcal{M}^{(1)}$, yields

$$
\mathrm{E}_{1} \leq C|I|^{1 / 2} \sum_{R \in m(\Omega)} \int_{x_{2} \notin 10 J^{\prime}}\left(\int_{x_{1} \in 10 I} \iint_{\Gamma\left(x_{2}\right)}\left|\psi_{t_{2}} *_{2} a_{R}\left(x_{1}, y_{2}\right)\right|^{2} \frac{d y_{2} d t_{2}}{t_{2}^{m+1}} d x_{1}\right)^{1 / 2} d x_{2} .
$$

By the similar argument to (3.7), one writes

$$
\left|\psi_{t_{2}} *_{2} a_{R}\left(x_{1}, y_{2}\right)\right| \leq \int_{\mathbb{R}^{m}}\left(1 \wedge \frac{\ell(J)}{t_{2}}\right) \frac{t_{2}^{1 / 2}}{\left(t_{2}+\left|x_{2}-x_{J}\right|\right)^{m+1 / 2}}\left|a_{R}\left(x_{1}, z_{2}\right)\right| d z_{2} .
$$

Substituting (3.9) back into (3.8) and then using Lemma 2.1 we can obtain

$$
\mathrm{E}_{1} \leq C \sum_{R \in m(\Omega)}|R|^{1 / 2}\left(\frac{\ell(J)}{\ell\left(J^{\prime}\right)}\right)^{1 / 2}\left\|a_{R}\right\|_{2} \leq C .
$$

Therefore, we obtain the required estimate (3.4), which implies (3.3).

Method 2 (via distribution inequality). It suffices to prove that

$$
\begin{aligned}
& \int_{\mathbb{R}^{n} \times \mathbb{R}^{m}} \sup _{\left|x_{1}-y_{1}\right|<t_{1}}\left(\iint_{\Gamma\left(x_{2}\right)}\left|P_{t_{1}} \psi_{t_{2}} * f\left(y_{1}, y_{2}\right)\right|^{2} \frac{d y_{2} d t_{2}}{t_{2}^{m+1}}\right)^{1 / 2} d x_{1} d x_{2} \\
& \leq C \int_{\mathbb{R}^{n} \times \mathbb{R}^{m}}\left(\iint_{\Gamma_{\beta}\left(x_{1}\right)} \iint_{\Gamma\left(x_{2}\right)}\left|t_{1} \nabla P_{t_{1}} \psi_{t_{2}} * f\left(y_{1}, y_{2}\right)\right|^{2} \frac{d y_{2} d t_{2}}{t_{2}^{m+1}} \frac{d y_{1} d t_{1}}{t_{1}^{n+1}}\right)^{1 / 2} d x_{1} d x_{2}
\end{aligned}
$$

for any $f \in H^{1}\left(\mathbb{R}^{n} \times \mathbb{R}^{m}\right) \cap L^{2}\left(\mathbb{R}^{n} \times \mathbb{R}^{m}\right)$, where $P_{t_{1}}$ is the Poisson kernel in $\mathbb{R}^{n}, C$ is a constant independent of $f$ and $\Gamma_{\beta}\left(x_{1}\right)=\left\{\left(y_{1}, t_{1}\right):\left|x_{1}-y_{1}\right|<\beta t_{1}\right\}$ is the cone with aperture $\beta>1$.

Now set $F_{t_{2}, y_{2}}\left(y_{1}\right)=\psi_{t_{2}} *_{2} f\left(y_{1}, y_{2}\right)$. Then we can further define the vectorvalued square function and nontangential maximal function of $F_{t_{2}, y_{2}}\left(y_{1}\right)$ as follows:

$$
\begin{aligned}
S_{\beta}(F)\left(x_{1}, x_{2}\right) & =\left(\iint_{\Gamma_{\beta}\left(x_{1}\right)}\left\|t_{1} \nabla P_{t_{1}} *_{1} F_{t_{2}, y_{2}}\left(y_{1}\right)\right\|_{\mathbb{B}\left(x_{2}\right)}^{2} \frac{d y_{1} d t_{1}}{t_{1}^{n+1}}\right)^{1 / 2}, \\
F^{*}\left(x_{1}, x_{2}\right) & =\sup _{\left|x_{1}-y_{1}\right|<t_{1}}\left\|P_{t_{1}} *_{1} F_{t_{2}, y_{2}}\left(y_{1}\right)\right\|_{\mathbb{B}\left(x_{2}\right)},
\end{aligned}
$$

where $\mathbb{B}\left(x_{2}\right)$ is as in (2.1).

Thus, to prove (3.10), it suffices to prove that

$$
\int_{\mathbb{R}^{n} \times \mathbb{R}^{m}} F^{*}\left(x_{1}, x_{2}\right) d x_{1} d x_{2} \leq C \int_{\mathbb{R}^{n} \times \mathbb{R}^{m}} S_{\beta}(F)\left(x_{1}, x_{2}\right) d x_{1} d x_{2} .
$$

We let $E$ be the closed set $\left\{x_{1} \in \mathbb{R}^{n}: S_{\beta}(F)\left(x_{1}, x_{2}\right) \leq \alpha\right\}$ and $A:=E^{c}$. Thus, $\lambda_{S_{\beta}(F)}(\alpha)=:\left|\left\{x_{1} \in \mathbb{R}^{n}: S_{\beta}(F)\left(x_{1}, x_{2}\right)>\alpha\right\}\right|=|A|$. Similarly, let $\lambda_{F^{*}}(\alpha)=$ $\left|\left\{x_{1} \in \mathbb{R}^{n}: F^{*}\left(x_{1}, x_{2}\right)>\alpha\right\}\right|$. 
To show (3.13), we will prove that for all $\alpha>0$ and a.e. $x_{2} \in \mathbb{R}^{m}$,

$$
\lambda_{F^{*}}(\alpha) \leq C\left\{\lambda_{S_{\beta}(F)}(\alpha)+\alpha^{-2} \int_{0}^{\alpha} s \lambda_{S_{\beta}(F)}(s) d s\right\} .
$$

Now let $E_{0}$ be those points at which $E$ has relative density at least $1 / 2$; more precisely, set $E_{0}=\left\{x_{1} \in \mathbb{R}^{n}\right.$ : for every cube $Q$, such that $x_{1} \in Q,|E \cap Q| \geq$ $\left.\frac{1}{2}|Q|\right\}$. Observe that since $E$ is closed, $E_{0} \subset E$; clearly $E_{0}$ is closed. If $\chi_{A}$ is the characteristic function of $A=E^{c}$, then $E_{0}^{c}=A^{*}=\left\{x_{1} \in \mathbb{R}^{n}: \mathcal{M}^{(1)}\left(\chi_{A}\right)\left(x_{1}, x_{2}\right)>\right.$ $1 / 2\}$. Thus $\left|A^{*}\right| \leq C|A|=C \lambda_{S_{\beta}(F)}(\alpha)$.

We now form the region $\mathcal{R}=\bigcup_{x_{1} \in E_{0}} \Gamma\left(x_{1}\right)$ with the corresponding approximating regions $\mathcal{R}_{\epsilon}$ whose boundaries, $\mathcal{B}_{\epsilon}$, are given as hypersurfaces $t=c \delta_{\epsilon}(x)$ with $\delta_{\epsilon}(x)$ smooth and $\left|\partial \delta_{\epsilon} / \partial x_{j}\right| \leq 1, j=1, \ldots, n$. (See [18, page 206.) We have

$$
\begin{aligned}
& \int_{E} S_{\beta}(F)\left(x_{1}, x_{2}\right)^{2} d x_{1} \\
& =\int_{\bigcup_{x_{1} \in E} \Gamma_{\beta}\left(x_{1}\right)}\left\|\nabla P_{t_{1}} *_{1} F_{t_{2}, y_{2}}\left(y_{1}\right)\right\|_{\mathbb{B}\left(x_{2}\right)}^{2}\left|\left\{x_{1} \in E:(y, t) \in \Gamma_{\beta}\left(x_{1}\right)\right\}\right| t_{1}^{1-n} d y_{1} d t_{1} .
\end{aligned}
$$

In the second integral we restrict integration over $(y, t) \in \mathcal{R}$, which implies that for some $\bar{x} \in E_{0},(y, t) \in \Gamma(\bar{x})$, i.e., $|\bar{x}-y|<t$. Then $(y, t) \in \Gamma_{\beta}\left(x_{1}\right)$ whenever $\left|x_{1}-\bar{x}\right|<(\beta-1) t$. Thus $\left|\left\{x_{1} \in E:(y, t) \in \Gamma_{\beta}\left(x_{1}\right)\right\}\right| \geq|E \cap B|$, where $B$ is the ball centered at $\bar{x} \in E_{0}$ with radius $(\beta-1) t$. In view of the definition of $E_{0}$, the latter quantity exceeds $c t^{n}$, and so

$$
\begin{aligned}
\int_{E} S_{\beta}(F)\left(x_{1}, x_{2}\right)^{2} d x_{1} & \geq C \int_{\mathcal{R}}\left\|\nabla P_{t_{1}} *_{1} F_{t_{2}, y_{2}}\left(y_{1}\right)\right\|_{\mathbb{B}\left(x_{2}\right)}^{2} t_{1} d y_{1} d t_{1} \\
& \geq C \int_{\mathcal{R}_{\epsilon}}\left\|\nabla P_{t_{1}} *_{1} F_{t_{2}, y_{2}}\left(y_{1}\right)\right\|_{\mathbb{B}\left(x_{2}\right)}^{2} t_{1} d y_{1} d t_{1} .
\end{aligned}
$$

We write the last integral as

$$
\begin{aligned}
& C \int_{\mathcal{R}_{\epsilon}} \iint_{\Gamma\left(x_{2}\right)}\left|\nabla P_{t_{1}} *_{1} F_{t_{2}, y_{2}}\left(y_{1}\right)\right|^{2} \frac{d y_{2} d t_{2}}{t_{2}^{m+1}} t_{1} d y_{1} d t_{1} \\
& =C \iint_{\Gamma\left(x_{2}\right)} \int_{\mathcal{R}_{\epsilon}}\left|\nabla P_{t_{1}} *_{1} F_{t_{2}, y_{2}}\left(y_{1}\right)\right|^{2} t_{1} d y_{1} d t_{1} \frac{d y_{2} d t_{2}}{t_{2}^{m+1}} .
\end{aligned}
$$

Then, transform the integral by Green's theorem, obtaining

$$
\begin{gathered}
\int_{E} S_{\beta}(F)\left(x_{1}, x_{2}\right)^{2} d x_{1} \geq C_{1} \iint_{\Gamma\left(x_{2}\right)} \int_{\mathcal{B}_{\epsilon}}\left|P_{t_{1}} *_{1} F_{t_{2}, y_{2}}\left(y_{1}\right)\right|^{2} d \sigma \frac{d y_{2} d t_{2}}{t_{2}^{m+1}} \\
-C_{2} \iint_{\Gamma\left(x_{2}\right)} \int_{\mathcal{B}_{\epsilon}}\left|P_{t_{1}} *_{1} F_{t_{2}, y_{2}}\left(y_{1}\right)\right| t_{1}\left|\nabla P_{t_{1}} *_{1} F_{t_{2}, y_{2}}\left(y_{1}\right)\right| d \sigma \frac{d y_{2} d t_{2}}{t_{2}^{m+1}},
\end{gathered}
$$

where $C_{1}$ and $C_{2}$ are two positive constants independent of $\epsilon$.

Let

$$
\mathcal{J}_{\epsilon}^{2}=\iint_{\Gamma\left(x_{2}\right)} \int_{\mathcal{B}_{\epsilon}}\left|P_{t_{1}} *_{1} F_{t_{2}, y_{2}}\left(y_{1}\right)\right|^{2} d \sigma \frac{d y_{2} d t_{2}}{t_{2}^{m+1}}=\int_{\mathcal{B}_{\epsilon}}\left\|P_{t_{1}} *_{1} F_{t_{2}, y_{2}}\left(y_{1}\right)\right\|_{\mathbb{B}\left(x_{2}\right)}^{2} d \sigma .
$$

We have

$$
\int_{\mathcal{B}_{\epsilon}}\left\|P_{t_{1}} *_{1} F_{t_{2}, y_{2}}\left(y_{1}\right)\right\|_{\mathbb{B}\left(x_{2}\right)}^{2} d \sigma \leq C \int_{\mathcal{B}_{\epsilon}}\left(F^{*}\right)^{2} d \sigma \leq C \int_{\mathbb{R}^{n}}\left(F^{*}\right)^{2} d x_{1}<\infty
$$

for a.e. $x_{2} \in \mathbb{R}^{m}$. Hence $\mathcal{J}_{\epsilon}$ is finite for every $\epsilon$. 
We now divide the boundary $\mathcal{B}_{\epsilon}$ into two parts $\mathcal{B}_{\epsilon}=\mathcal{B}_{\epsilon}^{E_{0}} \cup \mathcal{B}_{\epsilon}^{B^{*}}$, where $\mathcal{B}_{\epsilon}^{E_{0}}$ is the part above the set $E_{0}$, and $\mathcal{B}_{\epsilon}^{B^{*}}$ is the part lying above the set $B^{*}$. Next,

$$
\begin{aligned}
& \iint_{\Gamma\left(x_{2}\right)} \int_{\mathcal{B}_{\epsilon}}\left|P_{t_{1}} *_{1} F_{t_{2}, y_{2}}\left(y_{1}\right)\right| t_{1}\left|\nabla P_{t_{1}} *_{1} F_{t_{2}, y_{2}}\left(y_{1}\right)\right| d \sigma \frac{d y_{2} d t_{2}}{t_{2}^{m+1}} \\
& =\iint_{\Gamma\left(x_{2}\right)}\left(\int_{\mathcal{B}_{\epsilon} B^{*}}+\int_{\mathcal{B}_{\epsilon}^{E_{0}}}\right)\left|P_{t_{1}} *{ }_{1} F_{t_{2}, y_{2}}\left(y_{1}\right)\right| t_{1}\left|\nabla P_{t_{1}} *_{1} F_{t_{2}, y_{2}}\left(y_{1}\right)\right| d \sigma \frac{d y_{2} d t_{2}}{t_{2}^{m+1}} .
\end{aligned}
$$

To estimate the above two terms, we recall the following result on harmonic functions.

Lemma 3.4 ([18]). Suppose $u$ is harmonic in $\Gamma_{\beta}^{k}$. If $\iint_{\Gamma_{\beta}^{k}}|\nabla u|^{2} y^{1-n} d x d y \leq 1$, then $|y \nabla u| \leq C$ in $\Gamma_{\alpha}^{h}$. Here $\Gamma_{\beta}^{k}$ and $\Gamma_{\alpha}^{h}$ are truncated cones with $\beta>\alpha, k>h . C$ is a constant depending only on $\alpha, \beta, h, k$ and the dimension $n$.

Thus, by using Lemma 3.4, we have that $t_{1}\left\|\nabla P_{t_{1}} *_{1} F_{t_{2}, y_{2}}\left(y_{1}\right)\right\|_{\mathbb{B}\left(x_{2}\right)} \leq C \alpha$ since $S_{\beta}(F)\left(x_{1}, x_{2}\right) \leq \alpha$ for $x_{1} \in E$. By Schwarz's inequality, we get that

$$
\begin{aligned}
& \iint_{\Gamma\left(x_{2}\right)} \int_{\mathcal{B}_{\epsilon}^{B^{*}}}\left|P_{t_{1}} *_{1} F_{t_{2}, y_{2}}\left(y_{1}\right)\right| t_{1}\left|\nabla P_{t_{1}} *_{1} F_{t_{2}, y_{2}}\left(y_{1}\right)\right| d \sigma \frac{d y_{2} d t_{2}}{t_{2}^{m+1}} \\
& \quad \leq C \mathcal{J}_{\epsilon} \alpha \sigma\left(\mathcal{B}_{\epsilon}^{B^{*}}\right)^{1 / 2} \leq C \mathcal{J}_{\epsilon} \alpha\left|B^{*}\right|^{1 / 2} \leq C \mathcal{J}_{\epsilon}\left(\alpha^{2} \lambda_{S_{\beta}(F)}(\alpha)\right)^{1 / 2} .
\end{aligned}
$$

Moreover, $\mathcal{I}_{\epsilon}=\iint_{\Gamma\left(x_{2}\right)} \int_{\mathcal{B}_{\epsilon}^{E_{0}}}\left|P_{t_{1}} *_{1} F_{t_{2}, y_{2}}\left(y_{1}\right)\right| t_{1}\left|\nabla P_{t_{1}} *_{1} F_{t_{2}, y_{2}}\left(y_{1}\right)\right| d \sigma \frac{d y_{2} d t_{2}}{t_{2}^{m+1}} \rightarrow 0$ as $\epsilon \rightarrow 0$. Hence (3.15) gives $\mathcal{J}_{\epsilon}^{2} \leq C \int_{E} S_{\beta}(F)\left(x_{1}, x_{2}\right)^{2} d x_{1}+C \mathcal{J}_{\epsilon}\left(\alpha^{2} \lambda_{S_{\beta}(F)}(\alpha)\right)^{1 / 2}+$ $C \mathcal{I}_{\epsilon}$ and therefore

$$
\mathcal{J}_{\epsilon}^{2} \leq C\left\{\int_{E} S_{\beta}(F)\left(x_{1}, x_{2}\right)^{2} d x_{1}+\alpha^{2} \lambda_{S_{\beta}(F)}(\alpha)\right\}
$$

for $\epsilon$ small enough.

Next, for each $\epsilon>0$, define a function $f^{\epsilon}$ on $\mathbb{R}^{n}$ by setting

$$
f^{\epsilon}\left(x_{1}\right)=C\left\|P_{\delta_{\epsilon}\left(x_{1}\right)} *_{1} F_{t_{2}, y_{2}}\left(x_{1}\right)\right\|_{\mathbb{B}\left(x_{2}\right)}+C \alpha \chi_{B^{*}}\left(x_{1}\right),
$$

where $\chi_{B^{*}}$ is the characteristic function of $B^{*}$, and $t=\delta_{\epsilon}\left(x_{1}\right)$ is the equation of the hypersurface $\mathcal{B}_{\epsilon}=\partial \mathcal{R}_{\epsilon}$. Let $U_{\epsilon}\left(x_{1}, t_{1}\right)$ be the Poisson integral of the function $f^{\epsilon}$, i.e., $U_{\epsilon}\left(x_{1}, t_{1}\right)=P_{t_{1}} * f^{\epsilon}\left(x_{1}\right)$. We claim that

$$
\left\|P_{t_{1}} *_{1} F_{t_{2}, y_{2}}\left(x_{1}\right)\right\|_{\mathbb{B}\left(x_{2}\right)} \leq C U_{\epsilon}\left(x_{1}, t_{1}\right), \quad\left(x_{1}, t_{1}\right) \in \mathcal{B}_{\epsilon} .
$$

In fact, choose $\beta^{*}$ satisfying $1<\beta^{*}<\beta$. Then there exists a positive constant $C$ such that $B^{\prime}\left(\left(y_{1}, t_{1}\right), C t_{1}\right) \subset \bigcap_{x_{0} \in E_{0}} \Gamma_{\beta^{*}}\left(x_{0}\right)$ whenever $\left(y_{1}, t_{1}\right) \in \mathcal{B}_{\epsilon}$, where $B^{\prime}\left(\left(y_{1}, t_{1}\right), C t_{1}\right)$ is the ball centered at $\left(y_{1}, t_{1}\right)$ with radius $C t_{1}$. Now from Lemma 3.4, we have $t_{1}\left\|\nabla P_{t_{1}} *_{1} F_{t_{2}, y_{2}}\left(y_{1}\right)\right\|_{\mathbb{B}\left(x_{2}\right)} \leq C \alpha$ whenever $\left(y_{1}, t_{1}\right) \in B^{\prime}$. Set $Q_{1}=\left(y_{1}, t_{1}\right)$ and for any distinct point $Q_{2} \in B^{\prime}$, we have $\left|Q_{1}-Q_{2}\right| \leq C t_{1}$. Moreover,

$$
\begin{aligned}
& \left\|P_{t_{1}} *_{1} F_{t_{2}, y_{2}}(\cdot)\left(Q_{1}\right)-P_{t_{1}} *_{1} F_{t_{2}, y_{2}}(\cdot)\left(Q_{2}\right)\right\|_{\mathbb{B}\left(x_{2}\right)} \\
& \quad \leq C t_{1} \sup _{Q^{\prime} \in B^{\prime}}\left\|P_{t_{1}} *{ }_{1} F_{t_{2}, y_{2}}(\cdot)\left(Q^{\prime}\right)\right\|_{\mathbb{B}\left(x_{2}\right)} \leq C \alpha \chi_{B^{*}}\left(y_{1}\right) .
\end{aligned}
$$


Set $S_{\epsilon}=\mathcal{B}_{\epsilon} \cap B^{\prime}$. Then

$$
\begin{aligned}
\left\|P_{t_{1}} *_{1} F_{t_{2}, y_{2}}\left(y_{1}\right)\right\|_{\mathbb{B}\left(x_{2}\right)} & =\left\|P_{t_{1}} *_{1} F_{t_{2}, y_{2}}(\cdot)\left(Q_{1}\right)\right\|_{\mathbb{B}\left(x_{2}\right)} \\
& \leq \frac{1}{\left|S_{\epsilon}\right|} \int_{S_{\epsilon}}\left\|P_{t_{1}} *_{1} F_{t_{2}, y_{2}}(\cdot)\left(Q_{2}\right)\right\|_{\mathbb{B}\left(x_{2}\right)} d \sigma+C \alpha \chi_{B^{*}}\left(y_{1}\right) \\
& \leq \frac{C}{t_{1}^{n}} \int_{\left|z-y_{1}\right|<C t_{1}}\left|f^{\epsilon}(z)\right| d z \\
& \leq C \int_{\left|z-y_{1}\right|<C t_{1}} \frac{t_{1}}{\left(t_{1}^{2}+\left|z-y_{1}\right|^{2}\right)^{\frac{n+1}{2}}}\left|f^{\epsilon}(z)\right| d z \\
& \leq C P_{t_{1}} *\left(f^{\epsilon}\right)\left(y_{1}\right)=U_{\epsilon}\left(y_{1}, t_{1}\right) .
\end{aligned}
$$

We then select a subsequence of the $f^{\epsilon}$ which converges weakly to $\bar{f} \in L^{2}\left(\mathbb{R}^{n}\right)$. From (3.16), we have

$$
\int_{\mathbb{R}^{n}}\left|\bar{f}\left(x_{1}\right)\right|^{2} d x_{1} \leq C\left\{\int_{E} S_{\beta}(F)\left(x_{1}, x_{2}\right)^{2} d x_{1}+\alpha^{2} \lambda_{S_{\beta}(F)}(\alpha)\right\} .
$$

Passing to the limit, we obtain that

$$
\left\|P_{t_{1}} *_{1} F_{t_{2}, y_{2}}\left(y_{1}\right)\right\|_{\mathbb{B}\left(x_{2}\right)} \leq C U\left(y_{1}, t_{1}\right), \quad\left(y_{1}, t_{1}\right) \in \mathcal{R}
$$

where $U$ is the Poisson kernel of $\bar{f}$, and $F^{*}\left(x_{1}\right) \leq U^{*}\left(x_{1}\right)$ for $x_{1} \in E_{0}$. Thus, we have

$$
\begin{aligned}
\lambda_{F^{*}}(\alpha) & \leq\left|E_{0}^{c}\right|+\left|\left\{x_{1} \in E_{0}: F^{*}\left(x_{1}\right)>\alpha\right\}\right| \\
& \leq C \lambda_{S_{\beta}(F)}(\alpha)+\alpha^{-2} \int_{E_{0}} F^{*}\left(x_{1}\right)^{2} d x_{1} \\
& \leq C \lambda_{S_{\beta}(F)}(\alpha)+\alpha^{-2} \int_{E_{0}} U^{*}\left(x_{1}\right)^{2} d x_{1} \\
& \leq C \lambda_{S_{\beta}(F)}(\alpha)+\alpha^{-2} \int_{E_{0}} \bar{f}\left(x_{1}\right)^{2} d x_{1} \\
& \leq C \lambda_{S_{\beta}(F)}(\alpha)+C \alpha^{-2} \int_{E} S_{\beta}(F)\left(x_{1}, x_{2}\right)^{2} d x_{1} \\
& \leq C \lambda_{S_{\beta}(F)}(\alpha)+C \alpha^{-2} \int_{0}^{\alpha} s \lambda_{S_{\beta}(F)}(s) d s,
\end{aligned}
$$

which implies (3.14).

Proof of Proposition 3.3. It suffices to prove that there exists a constant $C>0$ such that

$$
\begin{aligned}
& \int_{\mathbb{R}^{n} \times \mathbb{R}^{m}}\left(\iint_{\Gamma\left(x_{1}\right)} \iint_{\Gamma\left(x_{2}\right)}\left|t_{1} \nabla P_{t_{1}} \psi_{t_{2}} * f\left(y_{1}, y_{2}\right)\right|^{2} \frac{d y_{2} d t_{2}}{t_{2}^{m+1}} \frac{d y_{1} d t_{1}}{t_{1}^{n+1}}\right)^{1 / 2} d x_{1} d x_{2} \\
& \quad \leq C \int_{\mathbb{R}^{n} \times \mathbb{R}^{m}} \sup _{\left|x_{1}-y_{1}\right|<t_{1}}\left(\iint_{\Gamma\left(x_{2}\right)}\left|P_{t_{1}} \psi_{t_{2}} * f\left(y_{1}, y_{2}\right)\right|^{2} \frac{d y_{2} d t_{2}}{t_{2}^{m+1}}\right)^{1 / 2} d x_{1} d x_{2}
\end{aligned}
$$

for any $f \in H_{\mathcal{N}, S}^{1}\left(\mathbb{R}^{n} \times \mathbb{R}^{m}\right) \cap L^{2}\left(\mathbb{R}^{n} \times \mathbb{R}^{m}\right)$.

Let $S_{\beta}(F)\left(x_{1}, x_{2}\right)$ and $F^{*}\left(x_{1}, x_{2}\right)$ be the same as (3.11) and (3.12), respectively. Set $S(F)\left(x_{1}, x_{2}\right)=S_{1}(F)\left(x_{1}, x_{2}\right)$. Thus, to prove (3.19), it suffices to prove that

$$
\int_{\mathbb{R}^{n} \times \mathbb{R}^{m}} S(F)\left(x_{1}, x_{2}\right) d x_{1} d x_{2} \leq C \int_{\mathbb{R}^{n} \times \mathbb{R}^{m}} F^{*}\left(x_{1}, x_{2}\right) d x_{1} d x_{2}
$$


To prove (3.20), it is enough to show that for all $\alpha>0$ and almost everywhere $x_{2} \in \mathbb{R}^{m}$,

$$
\begin{aligned}
& \int_{\left\{\mathcal{M}^{(1)}\left(\chi_{F^{*}>\alpha}\right)\left(x_{1}\right)<2^{-(n+1)}\right\}} S(F)\left(x_{1}, x_{2}\right)^{2} d x_{1} \\
& \quad \leq C \alpha^{2}\left|\left\{x_{1}: F^{*}\left(x_{1}, x_{2}\right)>\alpha\right\}\right|+C \int_{\left\{x_{1}: F^{*}\left(x_{1}, x_{2}\right) \leq \alpha\right\}} F^{*}\left(x_{1}, x_{2}\right)^{2} d x_{1} .
\end{aligned}
$$

In fact, note that for almost everywhere $x_{2} \in \mathbb{R}^{m}$, we have

$$
\int_{\mathbb{R}^{n}} S(F)\left(x_{1}, x_{2}\right) d x_{1}=\int_{0}^{\infty}\left|\left\{x_{1}: S(F)\left(x_{1}, x_{2}\right)>\alpha\right\}\right| d \alpha .
$$

Suppose (3.21) holds. Then

$$
\begin{aligned}
\left|\left\{x_{1}: S(F)\left(x_{1}, x_{2}\right)>\alpha\right\}\right| \leq & \left|\left\{x_{1}: S(F)\left(x_{1}, x_{2}\right)>\alpha, \mathcal{M}^{(1)}\left(\chi_{F^{*}>\alpha}\right)\left(x_{1}\right)<2^{-(n+1)}\right\}\right| \\
& +\left|\left\{x_{1}: \mathcal{M}^{(1)}\left(\chi_{F^{*}>\alpha}\right)\left(x_{1}\right) \geq 2^{-(n+1)}\right\}\right| \\
\leq & \frac{1}{\alpha^{2}} \int_{\left\{\mathcal{M}^{(1)}\left(\chi_{F^{*}>\alpha}\right)\left(x_{1}\right)<2^{-(n+1)}\right\}} S(F)\left(x_{1}, x_{2}\right)^{2} d x_{1} \\
& +C 2^{(n+1)}\left|\left\{x_{1}: F^{*}\left(x_{1}, x_{2}\right)>\alpha\right\}\right| \\
\leq & C \frac{1}{\alpha^{2}} \int_{\left\{x_{1}: F^{*}\left(x_{1}, x_{2}\right) \leq \alpha\right\}} F^{*}\left(x_{1}, x_{2}\right)^{2} d x_{1} \\
& +C\left|\left\{x_{1}: F^{*}\left(x_{1}, x_{2}\right)>\alpha\right\}\right| .
\end{aligned}
$$

By integrating $\alpha$ and $x_{2}$ on both sides of the inequality above, we then obtain (3.20).

Now let us prove 3.21):

$$
\begin{aligned}
& \int_{\left\{\mathcal{M}^{(1)}\left(\chi_{F^{*}>\alpha}\right)\left(x_{1}\right)<2^{-(n+1)}\right\}} S(F)\left(x_{1}, x_{2}\right)^{2} d x_{1} \\
& =\iint_{\Gamma\left(x_{2}\right)} \int_{\left\{\mathcal{M}^{(1)}\left(\chi_{F^{*}>\alpha}\right)\left(x_{1}\right)<2^{-(n+1)}\right\}} \iint_{\Gamma\left(x_{1}\right)}\left|t_{1} \nabla P_{t_{1}} *_{1} F_{t_{2}, y_{2}}\left(y_{1}\right)\right|^{2} d x_{1} \frac{d y_{1} d t_{1}}{t_{1}^{n+1}} \frac{d y_{2} d t_{2}}{t_{2}^{m+1}} .
\end{aligned}
$$

Note that

$$
\begin{aligned}
& \left.\iint_{\left\{\mathcal{M}^{(1)}\left(\chi_{F^{*}>\alpha}\right)\left(x_{1}\right)<2^{-(n+1)}\right\}}\left|\int_{\Gamma\left(x_{1}\right)}\right| t_{1} \nabla P_{t_{1}} *_{1} F_{t_{2}, y_{2}}\left(y_{1}\right)\right|^{2} \frac{d y_{1} d t_{1}}{t_{1}^{n+1}} d x_{1} \\
& =\int_{R^{*}}\left|\nabla P_{t_{1}} *_{1} F_{t_{2}, y_{2}}\left(y_{1}\right)\right|^{2} t_{1}^{1-n}\left|B\left(y_{1}, t_{1}\right) \cap\left\{\mathcal{M}^{(1)}\left(\chi_{F^{*}>\alpha}\right)\left(x_{1}\right)<2^{-(n+1)}\right\}\right| d y_{1} d t_{1} \\
& \leq \int_{R^{*}}\left|\nabla P_{t_{1}} *_{1} F_{t_{2}, y_{2}}\left(y_{1}\right)\right|^{2} t_{1} d y_{1} d t_{1},
\end{aligned}
$$

where we use $B\left(y_{1}, t_{1}\right)$ to denote the ball in $\mathbb{R}^{n}$ centered at $y_{1}$ with radius $t_{1}$, and

$$
R^{*}=\left\{\left(y_{1}, t_{1}\right):\left|B\left(y_{1}, t_{1}\right) \cap\left\{z: F^{*}\left(z, x_{2}\right)>\alpha\right\}\right| \leq 2^{-(n+1)}\left|B\left(y_{1}, t_{1}\right)\right|\right\} .
$$

Thus, we have obtained that

$$
\begin{aligned}
& \int_{\left\{\mathcal{M}^{(1)}\left(\chi_{F^{*}>\alpha}\right)\left(x_{1}\right)<2^{-(n+1)}\right\}} S(F)\left(x_{1}, x_{2}\right)^{2} d x_{1} \\
& \leq \int_{R^{*}}\left\|\nabla P_{t_{1} *_{1}} F_{t_{2}, y_{2}}\left(y_{1}\right)\right\|_{\mathbb{B}\left(x_{2}\right)}^{2} t_{1} d y_{1} d t_{1} .
\end{aligned}
$$


It is easy to check that if $\left|B\left(y_{1}, t_{1}\right) \cap\left\{z: F^{*}\left(z, x_{2}\right)>\alpha\right\}\right| \leq 2^{-(n+1)}\left|B\left(y_{1}, t_{1}\right)\right|$, then $g * \Phi_{t_{1}}\left(y_{1}\right)>C$ for some constant $C>0$, where $\Phi \in C_{0}^{1}\left(\mathbb{R}^{n}\right)$ is as in Lemma 2.3 and $g(x)=\chi_{\left\{F^{*}\left(x, x_{2}\right) \leq \alpha\right\}}(x)$. This, together with Lemma 2.3] implies

$$
\begin{aligned}
& \int_{\left\{\mathcal{M}^{(1)}\left(\chi_{F^{*}>\alpha}\right)\left(x_{1}\right)<2^{-(n+1)}\right\}} S(F)\left(x_{1}, x_{2}\right)^{2} d x_{1} \\
& \leq C \int_{R^{*}}\left\|\nabla P_{t_{1}} *{ }_{1} F_{t_{2}, y_{2}}\left(y_{1}\right)\right\|_{\mathbb{B}\left(x_{2}\right)}^{2}\left|\Phi_{t_{1}} * g\left(y_{1}\right)\right| t_{1} d y_{1} d t_{1} \\
& \leq C \iint_{\Gamma\left(x_{2}\right)} \int_{\mathbb{R}_{+}^{n+1}}\left|\nabla P_{t_{1}} *{ }_{1} F_{t_{2}, y_{2}}\left(y_{1}\right)\right|^{2}\left|\Phi_{t_{1}} * g\left(y_{1}\right)\right| t_{1} d y_{1} d t_{1} \frac{d y_{2} d t_{2}}{t_{2}^{m+1}} \\
& \leq C \iint_{\Gamma\left(x_{2}\right)} \int_{\mathbb{R}^{n}}\left|F_{t_{2}, y_{2}}\left(x_{1}\right)\right|^{2}\left|g\left(x_{1}\right)\right|^{2} d x_{1} \frac{d y_{2} d t_{2}}{t_{2}^{m+1}} \\
& \quad+C \iint_{\Gamma\left(x_{2}\right)} \int_{\mathbb{R}_{+}^{n+1}}\left|P_{t_{1}} *_{1} F_{t_{2}, y_{2}}\left(y_{1}\right)\right|^{2}\left|\Psi_{t_{1}} * g\left(y_{1}\right)\right|^{2} \frac{d y_{1} d t_{1}}{t_{1}} \frac{d y_{2} d t_{2}}{t_{2}^{m+1}} \\
& =C \int_{\mathbb{R}^{n}}\left\|F_{t_{2}, y_{2}}\left(x_{1}\right)\right\|_{\mathbb{B}\left(x_{2}\right)}^{2}\left|g\left(x_{1}\right)\right|^{2} d x_{1} \\
& \quad+C \int_{\mathbb{R}_{+}^{n+1}}\left\|P_{t_{1}} *_{1} F_{t_{2}, y_{2}}\left(y_{1}\right)\right\|_{\mathbb{B}\left(x_{2}\right)}^{2}\left|\Psi_{t_{1}} * g\left(y_{1}\right)\right|^{2} \frac{d y_{1} d t_{1}}{t_{1}} \\
& =: \mathrm{I}+\mathrm{II},
\end{aligned}
$$

where the third inequality follows from Lemma 2.3

For the term I, from the definitions of $g(x)$ and $F^{*}\left(x_{1}, x_{2}\right)$, we have

$$
\mathrm{I} \leq C \int_{\left\{x_{1}: F^{*}\left(x_{1}, x_{2}\right) \leq \alpha\right\}} F^{*}\left(x_{1}, x_{2}\right)^{2} d x_{1} .
$$

As for the term II, we only need to consider $\Psi_{t_{1}} *(g)\left(y_{1}\right) \neq 0$. In this case, $B\left(y_{1}, t_{1}\right) \cap\left\{z_{1}, F^{*}\left(z_{1}, x_{2}\right) \leq \alpha\right\} \neq \emptyset$. Thus, there exists a point $z_{1}^{0} \in \mathbb{R}^{n}$ such that $\left|z_{1}^{0}-y_{1}\right|<t_{1}$ and $F^{*}\left(z_{1}^{0}, x_{2}\right) \leq \alpha$. By the definition of $F^{*}\left(z_{1}^{0}, x_{2}\right)$, we have

$$
\left\|P_{t_{1} *_{1}} F_{t_{2}, y_{2}}\left(y_{1}\right)\right\|_{\mathbb{B}\left(x_{2}\right)} \leq \sup _{\left|z_{1}^{0}-z_{1}\right|<s_{1}}\left\|P_{s_{1}} *_{1} F_{t_{2}, y_{2}}\left(z_{1}\right)\right\|_{\mathbb{B}\left(x_{2}\right)}=F^{*}\left(z_{1}^{0}, x_{2}\right) \leq \alpha .
$$

Therefore, by (3.23) and the cancellation condition of $\Psi$, we obtain

$$
\begin{aligned}
\mathrm{II} & \leq C \alpha^{2} \int_{\mathbb{R}_{+}^{n+1}}\left|\Psi_{t_{1}} * \chi_{F^{*}\left(\cdot, x_{2}\right) \leq \alpha}\left(y_{1}\right)\right|^{2} \frac{d y_{1} d t_{1}}{t_{1}} \\
& =C \alpha^{2} \int_{\mathbb{R}_{+}^{n+1}}\left|\Psi_{t_{1}} * \chi_{F^{*}\left(\cdot, x_{2}\right)>\alpha}\left(y_{1}\right)\right|^{2} \frac{d y_{1} d t_{1}}{t_{1}} \\
& \leq C \alpha^{2}\left|\left\{y_{1}: F^{*}\left(y_{1}, x_{2}\right)>\alpha\right\}\right| .
\end{aligned}
$$

Combining the estimates of I and II, we can see that (3.21) holds, which implies that (3.20) holds. This completes the proof of Theorem 1.1 .

\section{Proof of Theorem 1.2}

4.1. Proof of $H^{1}\left(\mathbb{R}^{n} \times \mathbb{R}^{m}\right) \simeq H_{\mathcal{N}, g}^{1}\left(\mathbb{R}^{n} \times \mathbb{R}^{m}\right)$. By the similar argument to Method 1 of Proposition 3.2, we can easily check that

$$
H^{1}\left(\mathbb{R}^{n} \times \mathbb{R}^{m}\right) \subset H_{\mathcal{N}, g}^{1}\left(\mathbb{R}^{n} \times \mathbb{R}^{m}\right) \quad \text { with } \quad\|f\|_{H_{\mathcal{N}, g}^{1}\left(\mathbb{R}^{n} \times \mathbb{R}^{m}\right)} \leq C\|f\|_{H^{1}\left(\mathbb{R}^{n} \times \mathbb{R}^{m}\right)} .
$$


To prove the converse part, it suffices to prove that

$$
\begin{aligned}
\iint\left(\int_{0}^{\infty} \int_{0}^{\infty}\left|\psi_{t_{1}} \psi_{t_{2}} * f\left(x_{1}, x_{2}\right)\right|^{2} \frac{d t_{2}}{t_{2}} \frac{d t_{1}}{t_{1}}\right)^{1 / 2} d x_{1} d x_{2} \\
\quad \leq C \iint_{\left|x_{1}-y_{1}\right|<t_{1}}\left(\int_{0}^{\infty}\left|\phi_{t_{1}} \psi_{t_{2}} * f\left(y_{1}, x_{2}\right)\right|^{2} \frac{d t_{2}}{t_{2}}\right)^{1 / 2} d x_{1} d x_{2} .
\end{aligned}
$$

Denote by $\mathcal{B}$ the vector-valued function space $\left\{F_{t_{2}, x_{2}}\left(y_{1}\right): y_{1} \in \mathbb{R}^{n}, t_{2} \in(0, \infty)\right.$, $\left.x_{2} \in \mathbb{R}^{m}\right\}$ with the norm

$$
\left\|F_{t_{2}, x_{2}}\left(y_{1}\right)\right\|_{\mathcal{B}}=\left(\int_{0}^{\infty}\left|F_{t_{2}}\left(y_{1}, x_{2}\right)\right|^{2} \frac{d t_{2}}{t_{2}}\right)^{1 / 2} .
$$

We will prove (4.24) by two steps. Firstly, we can prove

$$
\begin{aligned}
& \iint\left(\iint_{\Gamma\left(x_{1}\right)}\left\|\psi_{t_{1}} \psi_{t_{2}} * f\left(y_{1}, x_{2}\right)\right\|_{\mathcal{B}}^{2} \frac{d y_{1} d t_{1}}{t_{1}^{n+1}}\right)^{1 / 2} d x_{1} d x_{2} \\
& \quad \leq C \iint_{\left|x_{1}-y_{1}\right|<t_{1}}\left(\sup _{0}^{\infty}\left\|\phi_{t_{1}} \psi_{t_{2}} * f\left(y_{1}, x_{2}\right)\right\|_{\mathcal{B}}^{2} \frac{d t_{1}}{t_{1}}\right)^{1 / 2} d x_{1} d x_{2} .
\end{aligned}
$$

In fact, the proof is similar to that of Proposition 3.3 with the minor modification that $\|\cdot\|_{\mathbb{B}\left(x_{2}\right)}$ is replaced by $\|\cdot\|_{\mathcal{B}}$.

Secondly, we claim that

$$
\left\|\left(\int_{0}^{\infty}|F(x, t)|^{2} \frac{d t}{t}\right)^{1 / 2}\right\|_{L^{1}\left(\mathbb{R}^{n}\right)} \leq C\left\|\left(\iint_{\Gamma(x)}|F(y, t)|^{2} \frac{d y d t}{t^{n+1}}\right)^{1 / 2}\right\|_{L^{1}\left(\mathbb{R}^{n}\right)}
$$

We observe that the right part of the inequality above is the $T_{2}^{1}$ norm of the function $F(x, t)$. By Proposition 2.2 we obtain $F(x, t)=\sum_{j} \lambda_{j} a_{j}(x, t)$, where $a_{j}(x, t)$ are $T_{2}^{1}$ atoms.

To prove (4.26), it is enough to prove that for each $T_{2}^{1}$ atom $a(x, t)$ associated to some cube $Q$, there exists a constant $C$ such that

$$
\left\|\left(\int_{0}^{\infty}|a(x, t)|^{2} \frac{d t}{t}\right)^{1 / 2}\right\|_{L^{1}\left(\mathbb{R}^{n}\right)} \leq C .
$$

In fact, by Hölder's inequality and the definition of the $T_{2}^{1}$ atom (see Section 2), we have

$$
\begin{gathered}
\int_{\mathbb{R}^{n}}\left(\int_{0}^{\infty}|a(y, t)|^{2} \frac{d t}{t}\right)^{1 / 2} d y=\int_{Q}\left(\int_{0}^{\ell(Q)}|a(y, t)|^{2} \frac{d t}{t}\right)^{1 / 2} d y \\
\leq\left(\iint_{\widehat{Q}}|a(y, t)|^{2} \frac{d y d t}{t}\right)^{1 / 2}|Q|^{1 / 2} \leq C .
\end{gathered}
$$

Therefore, we get (4.26). Let $F=\left(\int_{0}^{\infty}\left|\psi_{t_{1}} \psi_{t_{2}} * f\left(x_{1}, x_{2}\right)\right|^{2} \frac{d t_{2}}{t_{2}}\right)^{1 / 2}$. Substituting $F$ back into (4.26), we have

$$
\begin{gathered}
\int_{\mathbb{R}^{n}}\left(\int_{0}^{\infty} \int_{0}^{\infty}\left|\psi_{t_{1}} \psi_{t_{2}} * f\left(x_{1}, x_{2}\right)\right|^{2} \frac{d t_{2}}{t_{2}} \frac{d t_{1}}{t_{1}}\right)^{1 / 2} d x_{1} \\
\leq C \int_{\mathbb{R}^{n}}\left(\iint_{\Gamma\left(x_{1}\right)} \int_{0}^{\infty}\left|\psi_{t_{1}} \psi_{t_{2}} * f\left(y_{1}, x_{2}\right)\right|^{2} \frac{d t_{2}}{t_{2}} \frac{d y_{1} d t_{1}}{t_{1}^{n+1}}\right)^{1 / 2} d x_{1} .
\end{gathered}
$$


Integrating $x_{2}$ on both sides of the inequality above, we obtain

$$
\begin{aligned}
\iint\left(\int_{0}^{\infty} \int_{0}^{\infty}\left|\psi_{t_{1}} \psi_{t_{2}} * f\left(x_{1}, x_{2}\right)\right|^{2} \frac{d t_{2}}{t_{2}} \frac{d t_{1}}{t_{1}}\right)^{1 / 2} d x_{1} d x_{2} \\
\leq C \iint\left(\iint_{\Gamma\left(x_{1}\right)}\left\|\psi_{t_{1}} \psi_{t_{2}} * f\left(y_{1}, x_{2}\right)\right\|_{\mathcal{B}}^{2} \frac{d y_{1} d t_{1}}{t_{1}^{n+1}}\right)^{1 / 2} d x_{1} d x_{2} .
\end{aligned}
$$

(4.25), together with (4.27), implies (4.24).

4.2. $H^{1}\left(\mathbb{R}^{n} \times \mathbb{R}^{m}\right) \simeq H_{S, \mathcal{N}}^{1}\left(\mathbb{R}^{n} \times \mathbb{R}^{m}\right)$. It is easy to see that $\|f\|_{H_{\mathcal{N}, S}^{1}\left(\mathbb{R}^{n} \times \mathbb{R}^{m}\right)} \leq$ $\|f\|_{H_{S, \mathcal{N}}^{1}\left(\mathbb{R}^{n} \times \mathbb{R}^{m}\right)}$, which, together with the Main Theorem, implies $H_{S, \mathcal{N}}^{1}\left(\mathbb{R}^{n} \times\right.$ $\left.\mathbb{R}^{m}\right) \subset H^{1}\left(\mathbb{R}^{n} \times \mathbb{R}^{m}\right)$.

The proof of the inclusion $H^{1}\left(\mathbb{R}^{n} \times \mathbb{R}^{m}\right) \subset H_{S, \mathcal{N}}^{1}\left(\mathbb{R}^{n} \times \mathbb{R}^{m}\right)$ can be obtained by the similar argument to that of Method 1 of Proposition 3.2 .

4.3. By Subsections 4.1 and 4.2, we can obtain all the equivalent characterizations of $H^{1}\left(\mathbb{R}^{n} \times \mathbb{R}^{m}\right)$ mentioned in Theorem 1.2 .

\section{ACKNOWLEDGMENTS}

The authors would like to thank Professor Yongsheng Han for helpful suggestions and discussions. The authors also express their gratitude to the referee for a careful reading of the manuscript, giving numerous valuable suggestions to improve its mathematical accuracy.

\section{REFERENCES}

[1] S-Y. A. Chang and R. Fefferman, The Calderón-Zygmund decomposition on product domains, Amer. J. Math., 104 (1982), 445-468. MR658542 (84a:42028)

[2] S-Y. A. Chang and R. Fefferman, Some recent developments in Fourier analysis and $H^{p_{-}}$ theory on product domains, Bull. Amer. Math. Soc. (N.S.), 12 (1985), 1-43. MR766959 (86g:42038)

[3] R.R. Coifman, Y. Meyer and E.M. Stein, Some new function spaces and their applications to harmonic analysis, J. Funct. Anal., 62 (1985), 304-335. MR791851 (86i:46029)

[4] R. Fefferman, Calderón-Zygmund theory for product domains: $H^{p}$ spaces, Proc. Nat. Acad. Sci. USA, 83 (1986), 840-843. MR828217(87h:42032)

[5] R. Fefferman, Multiparameter Fourier analysis, Beijing Lecture in Harmonic Analysis, edited by E.M. Stein, 47-130, Princeton Univ. Press, Princeton, NJ, 1986. MR864371 (89a:42001)

[6] C. Fefferman and E.M. Stein, $H^{p}$ spaces of several variables, Acta Math., 129 (1972), 137-193. MR 0447953 (56:6263)

[7] R. Gundy and E.M. Stein, $H^{p}$ theory for the poly-disc, Proc. Nat. Acad. Sci., 76 (1979), 1026-1029. MR524328 (80j:32012)

[8] Y.S. Han, M.Y. Lee, C.C. Lin and Y.C. Lin, Calderón-Zygmund operators on product Hardy spaces, J. Funct. Anal., 258 (2010), 2834-2861. MR2593346

[9] Y.S. Han, G.Z. Lu and K. Zhao, Discrete Calderón's identity, atomic decomposition and boundedness criterion of operators on multiparameter Hardy spaces, J. Geom. Anal., 20 (2010), 670-689. MR2610894

[10] J.L. Journé, A covering lemma for product spaces, Proc. Amer. Math. Soc., 96 (1986), 593598. MR826486(87g:42028)

[11] J.L. Journé, Calderón-Zygmund operators on product spaces, Rev. Mat. Iberoamericana, 1 (1985), 55-91. MR836284 (88d:42028)

[12] J.L. Journé, Two problems of Calderón-Zygmund theory on product spaces, Ann. Inst. Fourier (Grenoble), 38 (1988), 111-132. MR 949001 (90b:42031)

[13] M. Lacey, S. Petermichl, J. Pipher and B. Wick, Multiparameter Riesz commutators, Amer. J. Math., 131 (2009), 731-769. MR2530853(2010d:42021) 
[14] K. Merryfield, On the area integral, Carleson measures and $H^{p}$ in the polydisc, Indiana Univ. Math. J., 34 (1985), 663-685. MR794581 (87c:42023)

[15] C. Muscalu, J. Pipher, T. Tao and C. Thiele, Bi-parameter paraproducts, Acta Math., 193 (2004), 269-296. MR2134868 (2005m:42028)

[16] C. Muscalu, J. Pipher, T. Tao and C. Thiele, Multi-parameter paraproducts, Rev. Mat. Iberoamericana, 22 (2006), 963-976. MR2320408 (2008b:42037)

[17] E.M. Stein, Harmonic analysis: Real variable methods, orthogonality and oscillatory integrals, Princeton Univ. Press, Princeton, NJ, 1993. MR,1232192 (95c:42002)

[18] E.M. Stein, Singular integrals and differentiability properties of functions, Princeton Univ. Press, Princeton, NJ, 1970. MR0290095 (44:7280)

Department of Mathematics, Sun Yat-sen University, Guangzhou, 510275, People's Republic of China

E-mail address: liji6@mail.sysu.edu.cn

Department of Mathematics, Sun Yat-sen University, Guangzhou, 510275, People's Republic of China

E-mail address: songl@mail.sysu.edu.cn

Department of Mathematics, Shantou University, Shantou, 515063, People's RepubLIC OF CHINA

E-mail address: cqtan@stu.edu.cn 